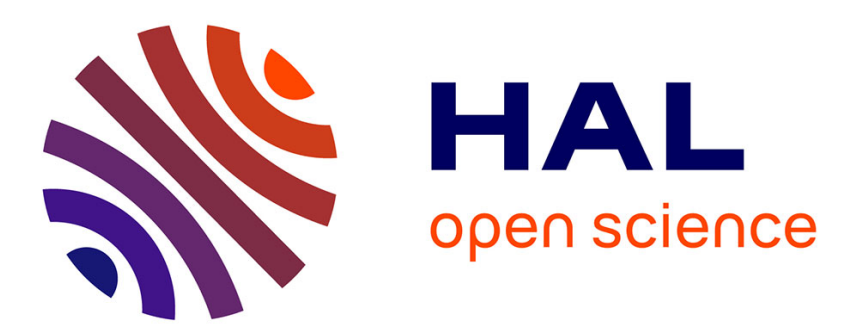

\title{
A Fine-grained Context-dependent Model for Indoor Spaces
}

\author{
Imad Afyouni, Cyril Ray, Christophe Claramunt
}

\section{To cite this version:}

Imad Afyouni, Cyril Ray, Christophe Claramunt. A Fine-grained Context-dependent Model for Indoor Spaces. Proceedings of the 2nd ACM SIGSPATIAL International Workshop on Indoor Spatial Awareness, Nov 2010, New York, United States. pp.33-38, 10.1145/1865885.1865894 . hal-00731165v2

\section{HAL Id: hal-00731165 \\ https://hal.science/hal-00731165v2}

Submitted on 12 Sep 2012

HAL is a multi-disciplinary open access archive for the deposit and dissemination of scientific research documents, whether they are published or not. The documents may come from teaching and research institutions in France or abroad, or from public or private research centers.
L'archive ouverte pluridisciplinaire HAL, est destinée au dépôt et à la diffusion de documents scientifiques de niveau recherche, publiés ou non, émanant des établissements d'enseignement et de recherche français ou étrangers, des laboratoires publics ou privés. 


\section{A Fine-grained Context-dependent Model for Indoor Spaces}

\author{
Imad Afyouni \\ Department of Computer \\ Science \\ Naval Academy Research \\ Institute \\ 29240 Brest Cedex 9, France \\ imad.afyouni@ecole-navale.fr
}

\author{
Cyril Ray \\ Department of Computer \\ Science \\ Naval Academy Research \\ Institute \\ 29240 Brest Cedex 9, France \\ cyril.ray@ecole-navale.fr
}

\author{
Christophe Claramunt \\ Department of Computer \\ Science \\ Naval Academy Research \\ Institute \\ 29240 Brest Cedex 9, France \\ claramunt@ecole-navale.fr
}

\begin{abstract}
The notion of context has been recently recognised as a key element in the development of context-aware information systems. Context varies according to application constraints, but also integrates the way users act in the environment and the interfaces they use to interact. Amongst many applications, indoor navigation systems strongly rely on contextbased information to adapt their services accordingly. Still, these systems need the design and development of flexible and dynamic spatial data models that will be able to provide appropriate data and services to mobile users acting in the environment. The preliminary research presented in this paper introduces a fine-grained and multi-layered spatial model that provides a flexible representation of an indoor space, and also takes into account the objects supported and acting in the environment. The modelling approach integrates different levels of granularity, from micro to macro levels, according to available positioning techniques, the infrastructure, device capabilities and user preferences.
\end{abstract}

\section{Categories and Subject Descriptors}

H.1 [Information Systems]: Models and Principles; E.1 [Data Structures]

\section{Keywords}

Indoor Spaces, Multi-level Spatial Modelling, Context-aware Information Systems

\section{INTRODUCTION}

The range of applications of geographical information science is progressively evolving from large to small scale environments. Amongst many factors, recent developments of ubiquitous computing and positioning techniques have largely favoured application of GIS to indoor spaces [10]. An indoor space can be informally defined as a built environment where people usually behave 13 (e.g., houses, commercial malls).
Indoor spaces are closely related to ambient systems where sensors provide real-time data without direct human interaction. A better modelling integration of indoor spaces and ambient systems still requires the development of appropriate spatial data structures and data manipulation facilities. We believe that this is a mandatory development for the delivery of intelligent-based context-aware systems applied to indoor spaces.

Structural approaches have long attempted to model indoor environments using graph-based representations as applied in space syntax 6], topological-based structures 7], [12], graphs by capturing the connectivity and accessibility between cellular units [8], and hierarchies [19], 18]. The main advantage of these approaches is that a structural representation allows the study of functional properties and the way humans behave in the environment. However, the geometry is not completely reflected, this introducing some bias in the analysis of the underlying spatial structure. This is why hybrid approaches that combine geometrical and structural properties appear as promising alternatives 9]. The main idea here is to combine a geometrical with a graph basedrepresentation, that is, a dual representation, implicitly hierarchical as those encompass knowledge of the environment at different levels of abstraction.

Clearly, integration of geometrical and structural representations favour integration of qualitative and quantitative points of view, this favouring a large spectrum of interacting representations and applications as suggested by the spatial semantic hierarchy initially introduced by Kuipers [11. This enables human reasoning and robot-based activities and even sensor-based interactions with the environment. Many approaches have adopted a more oriented sensor-based point of view when the objective is mostly oriented to the tracking and monitoring of mobile objects 8], 2], [14]. For instance, the model presented in 2] describes the space as a set of layers, the topographic space and the sensor space, and establishes connections between layers based on the sensor coverage. Deployment graphs were proposed in 8. by using different types of positioning sensors in order to improve indoor tracking accuracy. Alternative solutions represent the environment using a number of polygonal cells generated by exact and approximate space decomposition techniques [15. 
Modelling an indoor space implies to take into account the large range of applications that can be developed, and the available positioning techniques. Indoor spaces are indeed rich environments, where monitoring, design and planning are amongst many application examples that can often be built at different levels of abstraction. Such a model should also support different location-based techniques and this at the right level of granularity. Furthermore, indoor spaces should support user-oriented services (e.g. pedestrian navigation) and interactions between the different features that compose the environment (e.g., mobile objects, sensors, and continuous phenomena).

The preliminary research presented in this paper introduces a context-dependent multi-level spatial model. The modelling approach is multi-level since it is defined at a fine level of abstraction from which coarser levels of abstraction are derived appropriately when necessary. The choice of an appropriate level of abstraction is application and context dependent, and take into account several constraints such as positioning techniques, infrastructure properties, user capabilities and preferences. The modelling approach is based and extends a grid-based framework suggested in a related work 14, and on a semantic-based representation of an indoor space 3. The former achieves a continuous representation of an indoor space while the later introduces an approach that annotates objects of interest in an indoor space with semantic information according to the properties of the environment. The main principle of this approach is that objects are associated to specific regions of space that encapsulate different properties. The advantage of this approach is that it qualifies the different roles played by the objects in the environment, those roles being spatially qualified.

Our approach is based on these modelling concepts, but while those are oriented to the modelling of static objects in an indoor space, we propose a modelling approach that also integrates mobile objects and phenomena in order to take into account the dynamic properties and interactions of the represented system. The remainder of the paper is organized as follows. Section 2 introduces the main principles of our modelling approach. Section 3 discusses the model while Section 4 gives some perspectives and concludes the paper.

\section{MODELLING APPROACH 2.1 Sensor-based positioning}

Nowadays, the techniques currently available for indoor positioning range from radio-based technologies (WLAN, RFID, and Bluetooth) to non-radio technologies (infrared and ultrasound) to inertial navigation systems (INS) [10]. One challenge for all these techniques is to achieve a precise positioning of a given mobile user, together with some contextaware data that integrate the location of predefined sensors, and more important, a sense of the environment in which users are acting. This leads us to not only locate the users in the environment, but also the sensors available, and the spatial structure underneath. As these systems should be real-time based, in order to take into account the fact that mobile users behave in the environment, there is a general agreement on the fact that there is so far no perfect technique which is capable of reaching the desired accuracy continuously.

With this in mind, many approaches have adopted a hybrid approach combining several positioning technologies. For instance, 16] proposed an infrastructure-less positioning system for both indoor and outdoor environments. This system combines GPS receivers with inertial sensors (MEMS) and an indoor map-matching algorithm. The interest of MEMS sensors is that they usually provide acceleration and angular velocity. The principle behind this approach is that user location data have to be provided by the sensors continuously. When GPS data are not available, this being the common case in indoor environments, inertial sensors give alternative location data. However, inertial sensors are error-prone that causes sharp performance degradation. This implies to integrate additional rectification processes.

Similar techniques have been introduced elsewhere still for the design of ubiquitous location-based systems. In particular, when using WLAN or RFID positioning techniques, an integrated approach combines one (or both) of them with an inertial navigation system (INS) 4], 5. On the one hand, INS helps to overcome the lack of coverage of radio-frequency (RF) signals when an insufficient number of WLAN antennas or active RFID tags are deployed in an indoor environment. On the other hand, when both technologies (INS/Wi-Fi or INS/RFID) are available, a more precise position measurement can be produced by applying an appropriate multisensor data fusion technique [17.

\subsection{A Fine-grained Multi-layered Model}

We introduce a modelling approach of an indoor-based system that takes into account different levels of spatial granularity, and this regarding not only the way sensors are deployed in the environment, but also considering the fact that different models of space may coexist. The approach developed is a multi-granular spatial representation of an indoor environment that can be integrated into a context-aware system architecture. The approach also takes into account the large range and trends of positioning techniques to offer a context-dependent spatial model that supports different applications. Basically, this conceptual approach integrates a spatial representation at different levels of granularity. This concept is embodied in a fine-grained multi-layered model that implicitly embeds different levels from micro to macro, from continuous to discrete structures of space (Fig 1). One assumption of this model is that a coarse-grained model can be derived from a finer grained representation depending on the application and context-aware constraints and capabilities. The approach assumes that a given user acting in an indoor space will be continuously located in real-time, using for instance a MEMS sensor, this providing a micro-based representation of this user. In contrast, and when WLAN or RFID positioning systems are deployed in the environment, a coarser level of granularity might be provided to locate users in the environment. Similarly, the spatial representation to consider in order to relate mobile users to the environment will be chosen appropriately by taking into account some application constraints and properties. For instance, if 


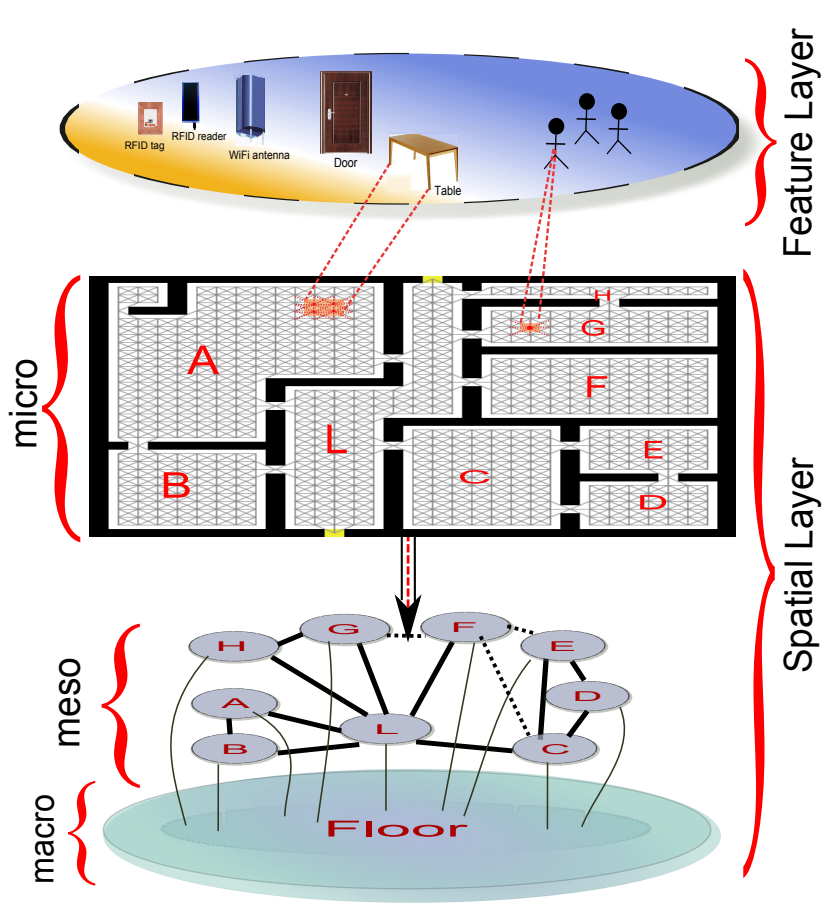

Figure 1: Multi-level representation of an indoor space and interactions between different layers

one wants to know how many users are located in a given room, it may be inappropriate to display the precise locations of those users and their trajectories. In such a case, a representation at the meso level is likely to be sufficient. Alternatively, a finer level of granularity might be appropriate when one is interested by the relative location of some users in a given room, and also with respect to the location of some sensors in that room.

The fine-grained spatial model represents an indoor environment with three complementary layers (Fig 2):

- The spatial layer organises the indoor space with a multigranular spatial model.

- The feature layer integrates the objects (i.e., mobile users, sensors, static objects) and continuous phenomena (e.g., noise diffusion, fire diffusion) of an indoor space.

- The action layer depicts how objects can interact and communicate in the environment.

The principle of this modelling approach is to make a difference between the features that compose the environment, their spatial representation, and the behaviours that emerge from them. Different levels of granularity are taken into account by the spatial layer, this favouring different functional and application requirements. The model extends and generalizes the notion of regions presented by 3 to be applied to all categories of features. Next, we describe in detail each of these layers and the intra- and inter-layer interaction pos-

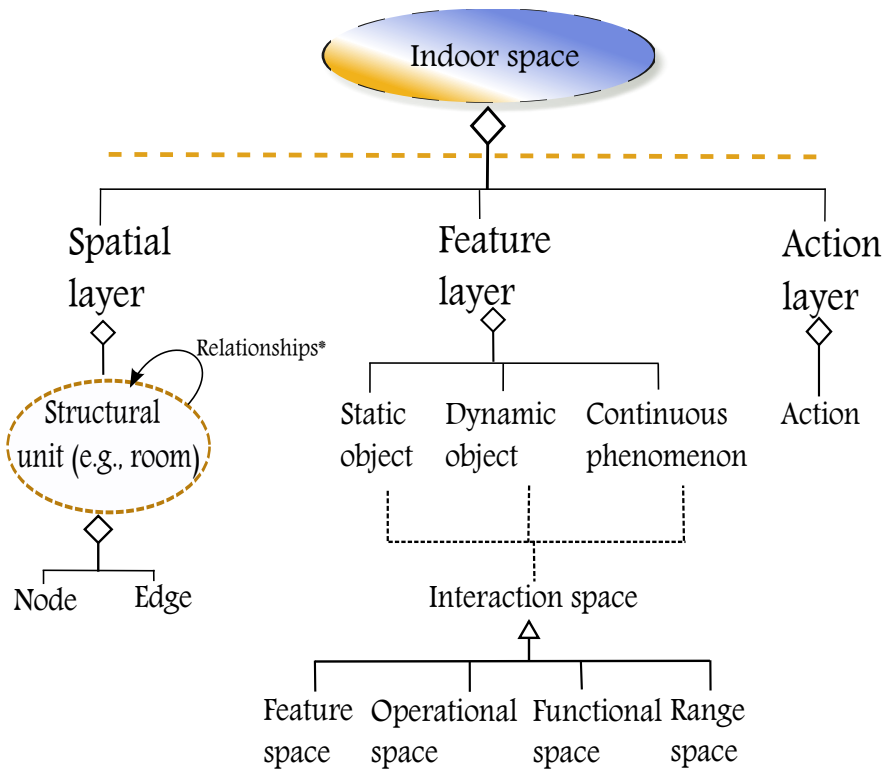

Figure 2: The Multi-layered model representing an indoor space. Interaction spaces represented as properties attached to each feature. Relationships between structural units include adjacencies, connections, etc.

sibilities.

Spatial layer

The spatial layer is made of a multi-level spatial representation. The principle behind is to consider an indoor space at a fine level of granularity. Depending on some application constraints, a coarser grained representation may be derived to best fit user needs and capabilities. This modelling concept can be materialized as a cell-based representation where each cell can represent some specific properties and constraints [16], and where the underlying space encapsulates a continuous representation of space that also constitutes a graphbased model of space as suggested in 14 .

One advantage of this modelling approach is that it achieves a maximum coverage of the indoor space. An indoor environment is represented as a continuous space that can support continuous positioning techniques used in indoor navigation. Also, the cell- and graph-based representation supports the modelling of structural properties (i.e., connections and relationships between nodes) at different levels of granularity, while keeping geometrical properties. Cells are considered as nodes by the graph-based representation, and are labelled according to their memberships to a given spatial unit such as a room or a connecting space. Therefore, each node has one and only one membership value since it belongs to one and only one spatial unit, whereas an edge might have multiple membership values when it intersects several spatial units. Nodes and edges can be labelled with impedances defined at the application level (i.e., node's and edge's accessibilities). At the meso level, Figure 1 illustrates the connectivity and adjacency relations between nodes of the coarse-grained rep- 


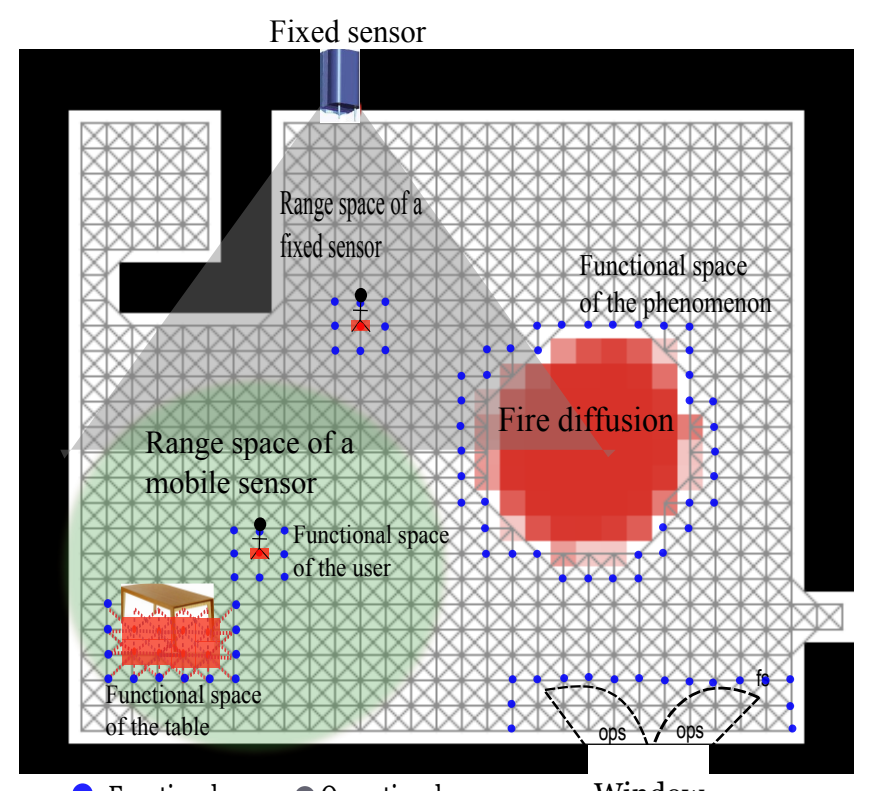

- Functional space Operational space

- Feature space

Figure 3: Interaction spaces

resentation. It is noteworthy to mention that the node $\mathrm{L}$ which is an abstraction of the corridor may be decomposed into several nodes by deploying additional partitioning sensors in order to achieve a more accurate representation.

As mentioned earlier, the level of granularity of the spatial representation can be chosen and displayed based on the application constraints and context-based information. Thanks to the flexibility offered by the spatial data structure, a coarser grained representation can be derived by aggregating nodes and edges according to some membership value, whereas edges will be derived accordingly. This favours data manipulation at a coarser level of granularity. For instance, structural queries can then be applied to find out emerging spatial and temporal properties and mobility behaviours at the level of the rooms and connections of an indoor space (e.g., objects located and passing through a given room for a period of time). Moreover, one can derive relationships between the sensors located in the environment and the resulting graph as suggested in [2] to achieve inter-layer interaction.

The modelled space is populated by a set of features of different types (static and dynamic objects, continuous phenomena). Regarding the modelling of continuous phenomena, the cell and graph spatial structure can support the diffusion of continuous properties as commonly done in cellularautomata modelling approaches 1 .

\section{Feature layer}

The feature layer models the objects and continuous phenomena of an indoor space that present an interest from an application point of view (Fig 2). These features are ei- ther attached to the infrastructure (i.e., static objects like tables, doors, walls, fixed sensors, ...) or evolving in the environment (i.e., mobile objects, continuous phenomena). We make a difference between features that represent a rigid entity (e.g., spatial objects, sensors, and users) and features that model a continuous phenomenon (e.g., gas leak or noise diffusion). By continuous feature we mean any kind of phenomenon that is likely to continuously spread over space. A feature may be discrete or continuous. A rigid entity may be static or dynamic and is modelled as an object. An object is identified, characterised by some properties (i.e., attributes) and the interaction spaces attached to it. An object has some static and potentially dynamic properties. In addition, an object can perform a selected list of actions that are triggered according to some contextual constraints which are application dependent. Each object is associated to several specific spaces that cover some semantic information that can be used for interaction purposes. At the finer level of granularity, an interaction space is made of a set of nodes and edges. We make a difference between the following interaction spaces (Fig 2):

- The feature space attached to a given feature is materialized by the nodes and edges covered by the physical object or continuous phenomenon.

- The operational space is materialised by the nodes and edges needed by the feature to perform its functions. It can be represented by the union of all potential nodes and edges an object may cover when performing its behaviour in the environment. For example, the operational space of a window comprises all potential nodes this window may cover when opening and closing.

- The functional space denotes the nodes and edges on which another feature can physically interact with the considered feature.

- The range space is a specific parameter assigned only to a sensor object and designates the set of nodes and edges covered by this sensor.

The different interaction spaces attached to the features are modelled when designing the fine-grained model of the environment.

Dynamic objects include mobile users equipped with mobile devices and mobile sensors (e.g., MEMS, RFID reader). Mobile users are characterized by their roles, preferences and purposes, and the capabilities of their devices. The notion of interaction spaces is generalized and applied on dynamic and continuous features as illustrated in Figure 3 . Let us consider the case of some users where the available positioning technique normally provides them with their estimated location. This location will be shifted to match the nearest node. Consequently we can say that:

- The feature space of a given user and at a given time henceforth refers to a given node based on the estimated location. - The operational space of a mobile user denotes the set of accessible nodes to the user at a given instant of time. It is largely dependent on the application and some contextualbased information. When considering mobile objects, the operational space is closely related to the properties and be- 
haviour encapsulated in the object. When considering continuous phenomena, it is closely related to the properties and the way the continuous phenomenon diffuses over space. Some examples that demonstrate how context information change the operational space of a mobile user:

First, context-based information largely depends on the user's profile. For instance, the operational space depends on whether the user is a fireman, wheelchair or normal user. Second, the concept of time (morning, night, weekend...) might have an impact when visiting a shopping centre. Third, we consider the case of a continuous phenomenon like gas leak or a fire that broke out inside a building. While the fire is spreading progressively within the space, a subset of nodes that are covered by the fire will be removed from the operational space. Instead, additional subsets which correspond to emergency exit routes will build up the new operational space. - The functional space refers to the interaction field around the physical space of the user, it is defined according to some specific user properties. In the example shown in Figure 3 a user is localised on a given node, so its functional space will comprise the subset of the eight neighbour nodes at a given instant.

- The range space is indeed the region covered by the mobile sensor which is either integrated in the mobile device or attached to the user.

One can remark that these modelling principles are applied to mobile objects as well as continuous phenomena. Interaction spaces are of different nature, and of different levels of granularity, this motivating further the multi-level modelling approach that supports different spatial representations. The different interaction spaces allow for different communication and interactions. For example two sensors or two users will be able to communicate and exchange some data when their operational spaces intersect.

\section{Action layer}

The action layer models the set of actions that a given feature can perform. When considering objects, actions specify whether and how objects of a given category change their states in order to behave in the environment. For instance, objects can adapt their behaviour and properties according to some contextual changes in the environment. So, actions denote the way objects interact with other objects and under which conditions, and how other objects can influence them. When considering a continuous phenomenon, actions can materialize the way a given phenomenon diffuses in space. The modelling approach implicitly leads to build semantic and topological relations amongst the set of features situated on the modelled space, by implicitly establishing relations between features, nodes and edges of different interaction spaces. In particular, applications of spatial and topological relationships are no longer limited to static objects but also to dynamic objects and continuous phenomena. Actions are thus context dependent. This means that at a given time and for a given feature, a selected list of actions are valid and can then be triggered according to some execution constraints. For instance, while navigating in an indoor space, users may ask for some direction and location information, a list of users interacting in the environment and in their neighbourhood, as well as checking the status of the features active in the environment. More typically, a given user can also ask for a complete coverage of the properties of a given space, or more specifically the ones that can physically interact with him. In fact, asynchronous physical interactions between two users can take place if one is located in the other's functional space. Moreover, a user can communicate with any fixed or mobile sensor located in the range space of a mobile sensor integrated in his device or attached to him.

\section{DISCUSSION}

A fine-grained partition of the indoor space is basically constructed with a suitable granularity that should approximate the physical space of the user. Hierarchical graph-based representations can then be derived with larger grains depending on the level of detail needed. One important parameter is the impedance value assigned to each node that helps to determine whether a node is accessible or not to a mobile user. For example, a node that is occupied at a given instant by some feature, is likely to have a high impedance value, thus, this node will be excluded from the operational space of the mobile user acting in the environment at that instant.

The number of nodes and edges encompassed in the spatial model as well as the number of features existing in the environment are source of increasing computational cost, and have to be managed in real time. The estimation process of the computational cost in both processing time and memory usage when constructing the fine grained spatial model is carried out in two phases: the set-up phase and the runtime phase. In the set-up phase, the entire graph-based map of the floor as well as the static objects that constitute the infrastructure are computed statically and stored in the user's device. The stored information include, for each node and edge, their identifiers and their corresponding properties, and for each static object, its properties and interaction spaces. At runtime, a mobile user may attempt to estimate its location and ask to communicate with another object by performing a scan of its environment. In this case, the interaction spaces of the user will be determined dynamically to respond in real time to the user request. The computational cost here is relatively limited. Moreover, triggering a continuous phenomenon may increase the computational load because it requires refreshing the system periodically so that it can update the interaction spaces of the users acting in the environment.

\section{CONCLUSION}

Spatial modelling is a key factor in the design and development of context-aware information systems applied to indoor spaces. One specific property of indoor spaces is that the spatial structure behind is as important as the different behaviours that compose the environment, whatever the level of granularity. In fact, indoor spaces should be ideally represented by a flexible spatial and dynamic data model that takes into account different spatial, structural and dynamic properties. The preliminary research presented in this paper introduces the principles of a fine-grained multi-layered space model. This approach assumes that an indoor environment can be modelled as three layers: The feature layer models the semantics of the objects and continuous phenomena located in a given indoor space. The spatial layer represents 
an indoor space with a multi-granular spatial representation. The action layer describes static and dynamic interactions between objects and phenomena in the environment. The model is flexible enough to support the development of different levels of data manipulation and interactions. We plan to apply the modelling approach to the monitoring of built environments and ships in terrestrial and maritime contexts, respectively.

\section{REFERENCES}

[1] M. Batty, Y. Xie, and Z. Sun. Modeling urban dynamics through gis-based cellular automata. Computers, Environment and Urban Systems, Elsevier, 23 (3):205-233, 1999.]

[2] T. Becker, C. Nagel, and T. Kolbe. Supporting Contexts for Indoor Navigation Using a Multilayered Space Model. In 2009 Tenth International Conference on Mobile Data Management: Systems, Services and Middleware, pages 680-685. IEEE, 2009. [

[3] M. Bhatt, F. Dylla, and J. Hois. Spatio-terminological Inference for the Design of Ambient Environments. Lecture Notes in Computer Science, 5756:371-391, 2009. [

[4] F. Evennou and F. Marx. Advanced integration of $\mathrm{WiFi}$ and inertial navigation systems for indoor mobile positioning. Eurasip Journal on Applied Signal Processing, (17):1-11, 2006.

[5] Q. Fu and G. Retscher. Using RFID and INS for Indoor Positioning. Location Based Services and Tele Cartography II, pages 421-438, 2009. [

[6] B. Hillier. Space is the Machine - A Configurational Theory of Architecture. Cambridge University Press, 1996. [

[7] H. Hu and D. Lee. Semantic location modeling for location navigation in mobile environment. In 2004 IEEE International Conference on Mobile Data Management, 2004. Proceedings, pages 52-61, 2004. [

[8] C. S. Jensen, H. Lu, and B. Yang. Graph model based indoor tracking. In Mobile Data Management, pages 122-131. IEEE Computer Society, 2009. [

[9] C. Jiang and P. Steenkiste. A hybrid location model with a computable location identifier for ubiquitous computing. Lecture notes in computer science, pages 246-263, 2002. [

[10] K. Kolodziej and J. Hjelm. Local positioning systems: LBS applications and services. CRC Press, 2006. \

[11] B. Kuipers. The Spatial Semantic Hierarchy. Artificial Intelligence, 119(1-2):191-233, 2000.

[12] D. Li and D. Lee. A topology-based semantic location model for indoor applications. In Proceedings of the 16th ACM SIGSPATIAL international conference on Advances in geographic information systems, pages 1-10. ACM, 2008. 』

[13] K.-J. Li. Indoor space: A new notion of space. In Proceedings of the 8th International Symposium on Web and Wireless Geographical Information Systems (W2GIS 2008), pages 1-3, 2008.

[14] X. Li, C. Claramunt, and C. Ray. A grid graph-based model for the analysis of 2D indoor spaces. Computers, Environment and Urban Systems, 2010.』

[15] M. Mekni and B. Moulin. Holonic Modelling of Large
Scale Geographic Environments. Lecture Notes in Computer Science, 5696:266, 2009.

[16] C. Ray, F. Comblet, J.-M. Bonnin, and Y.-M. Le Roux. Wireless and information technologies supporting intelligent location-based services. Wireless Technologies in Intelligent Transportation Systems, pages 225-265, 2010.]

[17] G. Retscher and Q. Fu. Integration of RFID, GNSS and DR for ubiquitous positioning in pedestrian navigation. Journal of Global Positioning Systems, 6(1):56-64, 2007.

[18] K. Richter, S. Winter, and U. Ruetschi. Constructing hierarchical representations of indoor spaces. In Proceedings of the 2009 Tenth International Conference on Mobile Data Management: Systems, Services and Middleware-Volume 00, pages 686-691. IEEE Computer Society, 2009. 』

[19] E. Stoffel, K. Schoder, and H. Ohlbach. Applying hierarchical graphs to pedestrian indoor navigation. In Proceedings of the 16th ACM SIGSPATIAL international conference on Advances in geographic information systems, page 54. ACM, 2008. [ 